\title{
Bradykinin B2 receptor and dopamine D2 receptor cooperatively contribute to the regulation of neutrophil adhesion to endothelial cells*
}

\author{
Anna Niewiarowska-Sendo', Anna Łabędź-Masłowska², Andrzej Kozik¹ and \\ Ibeth Guevara-Lora ${ }^{1 凶}$
}

1Department of Analytical Biochemistry, Faculty of Biochemistry, Biophysics and Biotechnology, Jagiellonian University in Krakow, Kraków, Poland; 2Department of Cell Biology, Faculty of Biochemistry, Biophysics and Biotechnology, Jagiellonian University in Krakow, Kraków, Poland

Leukocyte adhesion to the vascular endothelium contributes to many immunological and inflammatory disorders. These processes have been shown to be mediated by bradykinin receptor type 2 (B2R) and dopamine receptor type 2 (D2R). In a previous study, we reported the formation of a B2R-D2R heterodimer, possibly altering cellular functions. Hence, in the present study, we examined the effect of co-activation of endothelial cells with B2R and D2R agonists on the interaction of these cells with neutrophils. Bradykinin, the main B2R agonist, significantly increased cell adhesion, and this effect was reversed when the endothelial cells were additionally cotreated with a selective D2R agonist, sumanirole. These results were dependent on the incubation time, showing an opposite tendency after prolonged stimulation. Significant changes in the expression of adhesion proteins, such as E-selectin and intercellular adhesion molecule 1 in endothelial cells were observed. Additionally, the cells preincubated with tumor necrosis factor-a showed decreased cell adhesion and IL-8 release after long incubation with both agonists. The modulation of cell adhesion by D2R and B2R seem to be mediated via STAT3 phosphorylation. In summary, this study demonstrated a protective role of $D 2 R$ in neutrophil-endothelial cell adhesion induced by bradykinin, especially in cytokinestimulated endothelial cells.

Key words: bradykinin, bradykinin receptor, dopamine receptor, endothelial cells, neutrophil adhesion

Received: 03 April, 2018; revised: 19 June, 2018; accepted: 28 June, 2018; available on-line: 27 August, 2018, 2018

e-mail: ibeth.guevara-lora@uj.edu.pl

*This topic was presented in part at the 45th Winter School of the Faculty of Biochemistry, Biophysics and Biotechnology, 9-14 February 2018, Zakopane, Poland.

Abbreviations: $\mathrm{BK}$, bradykinin; $\mathrm{B} 1 \mathrm{R}$, bradykinin receptor type 1 $B 2 R$, bradykinin receptor type $2 ; D 2 R$, dopamine receptor type 2 ; GPCRs, G protein-coupled receptors; HOE 140 , icatibant; ICAM-1, intercellular adhesion molecule 1; IL-8, interleukin-8; PMN, polymorphonuclear cells; STAT3, signal transducer and activator of transcription 3; SUM, sumanirole; TNF-a, tumor necrosis factor alpha; VCAM-1, vascular cell adhesion molecule 1

\section{INTRODUCTION}

Initial stages of the inflammatory response of a vascularized tissue to various cellular lesions involve leukocyte recruitment, adhesion to the endothelium, and migration to the affected region. During these events, numerous molecular interactions occur between specific proteins that are present in the membranes of both endothelial cells and leukocytes (Langer \& Chavakis, 2009). Leukocyte recruitment is initiated by several chemokines, especially interleukin-8 (IL-8), and is followed by leukocyte rolling along the vascular surface, which engages a group of adhesion proteins called selectins. In turn, firm adhesion and sticking of leukocytes to the endothelium takes place via an interaction between leukocyte integrins and proteins of the endothelial cell surface, such as the intercellular and vascular cell adhesion molecules 1 (ICAM-1 and VCAM-1, respectively). Next, adherent leukocytes start migration from the vascular wall into the interstitial space, where they release proteolytic enzymes, oxygen free radicals, and other factors to destroy the inducers of the inflammatory response. All these processes are strictly regulated by proinflammatory cytokines produced by both leukocytes and endothelial cells, including tumor necrosis factor $\alpha(\mathrm{TNF}-\alpha)$ and interleukin- 1 , which up-regulate the expression of different adhesion molecules in cells (Langer \& Chavakis, 2009).

Additional substances that accumulate at the injury site during inflammation, such as kinin peptides play an important role in leukocyte adhesion (Guevara-Lora et al., 2011; Figueroa et al., 2015). Kinins, including bradykinin (BK), kallidin, and their metabolites without arginine at the C-end, are well known pro-inflammatory peptides, and are recognized by specific receptors bradykinin receptor type 1 and type 2 (B1R and B2R, respectively). These receptors belong to a large superfamily of $G$ protein-coupled receptors (GPCRs). DesArg metabolites activate mainly B1R, while BK and kallidin exert their biological effects via B2R (Bhoola et al., 1992; Leeb-Lundberg et al., 2005). Activation of B2R is particularly essential for the regulation of vascular tone and permeability, which control arterial pressure and cell migration through microvessel walls (Ishihara et al., 2002; Su, 2015). BK has been shown to increase monocyte adhesion to endothelial cells by enhancement of the expression of adhesion proteins, ICAM-1 and macrophage-1 antigen (Mac-1, CD11b/CD18) respectively, in human endothelial cells and monocytes (Guevara-Lora et al., 2014). In contrast, a reduced amount of rolling and adherent leukocytes was observed in mice with induced global cerebral ischemia treated with a B2R antagonist (Lehmberg et al., 2003). Moreover, the participation of $\mathrm{BK}$ in induction of IL-8 production by lung fibroblasts and human airway smooth muscle cells has also been documented (Koyama et al., 2000; Roscioni et al., 2009).

Other receptors belonging to the GPCR family as well as the dopamine receptors are abundantly expressed in the cardiovascular system, where they regulate vasodila- 
tation (Missale et al., 1998). These receptors are classified into two major groups: the D1 and D2 classes. The former group includes subtypes D1 and D5 while the latter one comprises subtypes D2, D3, and D4. They differ in their ability to modulate the production of cAMP. D1class receptors stimulate the production of cAMP, while receptors from the D2 class negatively regulate cAMP production. The dopamine receptor type 2 (D2R) has been demonstrated to be broadly involved in the regulation of anti-inflammatory responses. D2R agonists have been reported to enhance the production of interleukin 10 , i.e. an anti-inflammatory cytokine, in human T-lymphocytes (Besser et al., 2005). By contrast, D2R silencing in mouse renal proximal tubule cells increased the expression of TNF- $\alpha$ and monocyte chemoattractant protein-1 levels, while the treatment with the D2R agonist abolished an angiotensin II-induced effect on the release of these proinflammatory factors (Zhang et al., 2012). Moreover, D2R stimulation increased the expression of endogenous antioxidants, which prevent endothelial cell apoptosis (Horke et al., 2007). The importance of this receptor in the down-regulation of von Willebrand factor secretion, resulting in reduced endothelial activation during inflammation, has been reported as well (Zarei et al., 2006).

Recently, numerous studies have been focused on cooperation between GPCRs, which may result in changes in ligand affinities and cell signaling pathways of each receptor, causing significant modifications of cellular processes (Ferre et al., 2014). A considerable number of interactions of B2R and D2R with other GPCRs, resulting in oligomeric complexes, has been recently reported (Guevara-Lora et al., 2016). Moreover, our group demonstrated for the first time the formation of a functional heterodimer between B2R and D2R, capable of modulating cell responses, such as the intracellular $\mathrm{Ca}^{2+}$ and cAMP concentration (Niewiarowska-Sendo et al., 2017). All these facts suggest that the interaction between B2R and $\mathrm{D} 2 \mathrm{R}$ can have a consequence in the regulation of neutrophil adhesion to endothelial cells. To support of this hypothesis, in the present study, we investigated the effects of co-treatment with $\mathrm{BK}$ and sumanirole (SUM), specific agonists for B2R and D2R, respectively, on processes involved in neutrophil-endothelial cell adhesion.

\section{MATERIALS AND METHODS}

Chemicals. BK was supplied by Bachem (Bubendorf, Switzerland). Antibiotics, antimycotics, cell culture medium, and fetal bovine serum (FBS) were purchased from Corning (Corning, NY). Cell Tracker Red CMTPX was supplied by Molecular Probes (Eugene, OR). Bacitracin, captopril, endothelial cell growth supplement, heparin, icatibant (HOE 140), 2-mercaptomethyl-3-guanidinoethylthiopropanoic acid, protease inhibitors, SUM maleate, $\mathrm{S}$-(-)-eticlopride hydrochloride (eticlopride), and other standard chemicals were obtained from Sigma-Aldrich (St Louis, MO). Human TNF- $\alpha$ was purchased from Thermo Fisher Scientific (Waltham, MA).

Antibodies. Rabbit polyclonal antibody against Eselectin (ab185698) was purchased from Abcam (Cambridge, England). Human antibodies against ICAM-1, VCAM-1, and E-selectin conjugated with phycoerythrin (322708, 305806, and 322606, respectively) were supplied by Biolegend (San Diego, CA). Rabbit monoclonal antibodies against ICAM-1, signal transducer and activator of transcription 3 (STAT3), and phospho-STAT3 (\#4915, \#4904, and \#9145, respectively) were obtained from Cell Signaling Technology (Danvers, MA). Mouse monoclonal antibody against $\beta$-actin (MAB8929) and goat polyclonal antibody against mouse or rabbit $\mathrm{IgG}$ conjugated with horseradish peroxidase (HAF007 and HAF008, respectively) were purchased from R\&D Systems (Minneapolis, MN).

Cell culture. The human endothelial cell line (HUVEC-C; ATCC ${ }^{\circledR}$ CRL-1730 ${ }^{\mathrm{TM}}$ ) purchased from ATCC (Manassas, VA) was cultured in F-12K medium (Kaighn's Modification of Ham's F-12 Medium) supplemented with $10 \% \mathrm{FBS}, 1 \mathrm{U} / \mathrm{ml}$ penicillin, $1 \mu \mathrm{g} / \mathrm{ml}$ streptomycin, $2.5 \mu \mathrm{g} / \mathrm{ml}$ amphotericin B, $50 \mu \mathrm{g} / \mathrm{ml}$ endothelial cell growth supplement, and $100 \mu \mathrm{g} / \mathrm{ml}$ heparin at $37^{\circ} \mathrm{C}$ in a humidified atmosphere containing of $5 \% \mathrm{CO}_{2}$. Twentyfour hours before the experiments, cells were starved of the growth factors and the cells were stimulated in F-12K medium suplemented with $1 \%$ FBS and kininase inhibitors $(10 \mu \mathrm{M}$ 2-mercaptomethyl-3-guanidinoethylthiopropanoic acid, $20 \mu \mathrm{M}$ captopril, and $500 \mu \mathrm{M}$ bacitracin).

Neutrophil adhesion to endothelial cells. Human polymorphonuclear cells (PMN) were isolated as described previously (Zawrotniak et al., 2017) from freshly EDTA-treated whole-blood samples obtained from the Regional Blood Donation Center (Krakow, Poland), which complies with the requisite of confidentiality assurance for human participants. Isolated PMN were suspended in RPMI-1640 medium and loaded with Cell Tracker Red CMTPX in RPMI for $30 \mathrm{~min}$ at $37^{\circ} \mathrm{C}$, following the manufacturer's instructions, and then placed on a monolayer of endothelial cells stimulated as described below. Detailed description of the cell adhesion analysis was provided elsewhere (Guevara-Lora et al., 2011).

Confluent endothelial cells seeded in a 96-well microplate were pretreated or not pretreated with $10 \mathrm{ng} / \mathrm{ml}$ TNF- $\alpha$ for 24 hours and subsequently stimulated with $100 \mathrm{nM} \mathrm{BK}, 100 \mathrm{nM}$ SUM or both agonists for 6, 12, and 24 hours. Because cellular adhesion is a dynamic process depending on many factors, e.g. the presence of chemoattractants, in this study, we decided to evaluate the adhesion of PMN to endothelial cells at different intervals of the incubation time (Mestas \& Ley, 2008). Some samples were additionally preincubated for 30 minutes with B2R or D2R antagonists $(10 \mu \mathrm{M}$ HOE 140 or $10 \mu \mathrm{M}$ eticlopride, respectively) before cell stimulation with agonists.

Flow cytometry analysis. Confluent endothelial cells were stimulated with $100 \mathrm{nM}$ BK, $100 \mathrm{nM}$ SUM, or both agonists for 6 hours, i.e. an optimal time for relocation of adhesion molecules to the cell membrane (Scholz et al., 1996). After the incubation, the cells were washed, detached, and incubated for $30 \mathrm{~min}$ at $4{ }^{\circ} \mathrm{C}$ with phycoerythrin-conjugated antibodies at dilutions: 1:125 for ICAM-1 and 1:150 for VCAM-1 and E-selectin. After washing and resuspension, the mean fluorescence of the labeled cells was analyzed in a flow cytometer (Becton Dickinson, Franklin Lakes, NJ). Nonspecific fluorescence was determined using an isotype-matched human IgG (409301, Biolegend).

Protein analysis by Western blotting. Confluent endothelial cells were treated with $100 \mathrm{nM} \mathrm{BK}$, $100 \mathrm{nM}$ SUM, or both agonists for 6 and 24 hours to determine ICAM-1 and E-selectin or for 15 minutes to measure STAT3 and STAT3 phosphorylated at Tyr-705 (pSTAT3) in the medium supplemented with kininase inhibitors. Thereafter, cell lysates were prepared with Laemmli lysis buffer ( $0.125 \mathrm{M}$ Tris- $\mathrm{HCl}, 20 \%$ glycerol, 4\% sodium dodecyl sulfate (SDS), $400 \mathrm{mM}$ dithiothreitol, 
$0.004 \%$ bromophenol blue, $\mathrm{pH}$ 6.8) supplemented with $5 \mathrm{mM}$ sodium fluoride and $1 \mathrm{mM}$ sodium orthovanadate. Equal volumes of each sample were subjected to SDS-PAGE (polyacrylamide gel electrophoresis) in 12\% gel. Resolved proteins were transferred to polyvinylidene difluoride membranes (Sigma-Aldrich) in Tris/glycine buffer $(25 \mathrm{mM}$ Tris, $192 \mathrm{mM}$ glycine, $20 \%$ methanol, $\mathrm{pH} 8.3$ ) for 1 hour. After overnight blocking with 5\% non-fat milk, the membrane was incubated for 24 hours at $4^{\circ} \mathrm{C}$ with primary antibodies at dilutions of $1: 100$ for anti-E-selectin, 1:500 for anti-ICAM-1, 1:1000 for antipSTAT3 and anti-STAT3, and 1:2000 for anti- $\beta$-actin. Next, after extensive washing, the membrane was incubated with the respective secondary antibody conjugated with horseradish peroxidase at a 1:1000 dilution. After one-hour incubation and extensive washing, enzymatic reaction was performed with Immobilon Western Chemiluminescent HRP Substrate (Sigma-Aldrich) in accordance with the manufacturer's instructions. $\beta$-Actin was analyzed as a housekeeping protein in every blot as a normalization control.

Determination of interleukin- 8 concentration by ELISA. Confluent endothelial cells were pretreated or not with $10 \mathrm{ng} / \mathrm{ml} \mathrm{TNF}-\alpha$ for 24 hours and subsequently stimulated for 6 hours with $100 \mathrm{nM} \mathrm{BK}, 100 \mathrm{nM}$ SUM, or both agonists. Some samples were additionally preincubated with $10 \mu \mathrm{M}$ HOE 140 or $10 \mu \mathrm{M}$ eticlopride for 30 minutes prior to the induction with receptor agonists. The interleukin- 8 concentration in the medium was determined using a specific ELISA kit (BD Biosciences) according to the manufacturer's instructions and normalized to the amount of sample protein detected with the Lowry method. Absorbance was measured with a PowerWave X-Select microplate reader (BioTek Instruments, Winooski, VT) at $450 \mathrm{~nm}$.

Statistical analysis. Statistical analysis was performed with the $\mathrm{R}$ environment ( $\mathrm{R}$ Core Team, 2016). Representative data from at least three experiments were expressed as mean values \pm S.D. Student's $t$-test was used for statistical comparisons of mean values, and data were considered significant at $p<0.01$ or $p<0.05$, depending on the analyzed samples.

\section{RESULTS}

\section{Co-treatment of endothelial cells with B2R and D2R agonists alters neutrophil adhesion}

The fact that kinin peptides induce leukocyte adhesion to endothelial cells is well known (Guevara-Lora et al., 2011; Guevara-Lora et al., 2014; Figueroa et al., 2015). In order to investigate the effect of D2R activation on $\mathrm{BK}$-induced adhesion, endothelial cells were stimulated with $100 \mathrm{nM}$ BK and $100 \mathrm{nM}$ SUM and co-stimulated with both agonists at the same concentration at different incubation time: 6,12 , and 24 hours. The choice of the agonist concentration was supported by information from earlier reports, in which $100 \mathrm{nM}$ BK was considered to represent a pathological level (Blais et al., 2000) and $100 \mathrm{nM}$ SUM, one of the most selective D2R agonists, was close to a EC50 range in which this substance exhibited efficient binding to D2 receptors on cell membranes (McCall et al., 2005). In addition, during optimization studies, different concentrations of agonists (1 nM and $100 \mathrm{nM}$ ) did not cause substantial differences in the interaction between PMN and endothelial cells (see supplementary results - Fig. S1 at www.actabp.pl). Specific inhibitors were used to avoid bradykinin degradation by
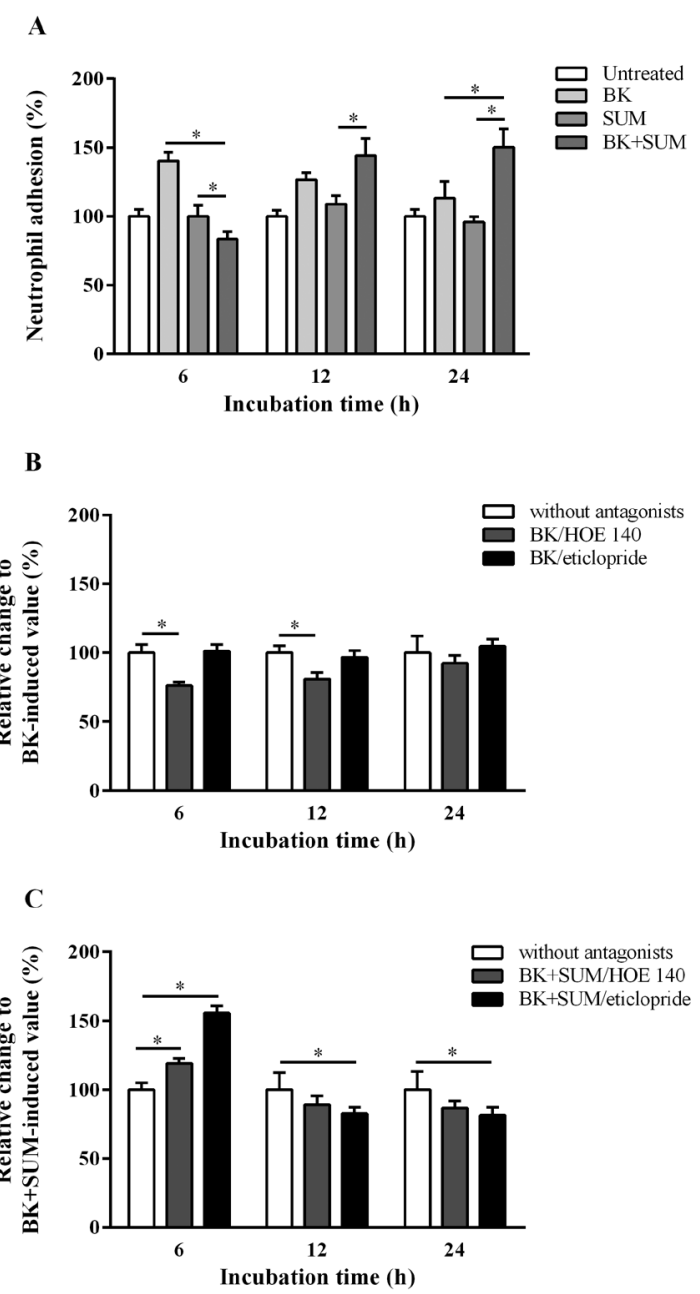

Figure 1. Neutrophil adhesion to endothelial cells treated with B2R and D2R agonists.

Labeled PMN were placed on confluent endothelial cells stimulated with $100 \mathrm{nM} \mathrm{BK}, 100 \mathrm{nM}$ SUM, or both agonists simultaneously for 6,12 , and 24 hours (A). Some samples were additionally preincubated with $10 \mu \mathrm{M}$ HOE 140 or $10 \mu \mathrm{M}$ eticlopride 30 minutes before receptor activation with the agonists $(\mathbf{B}, \mathbf{C})$. The bars represent the mean percentage of PMN adhesion to endothelial cells in relation to: (A) untreated cells, (B) BK-treated cells at the corresponding time, and (C) BK+SUM-treated cells at the corresponding time, assumed to have values equal to $100 \%$. In each case, at least three experiments were performed in triplicates. ${ }^{*} p<0.05$ between the indicated samples.

kininases. The results of the adhesion assay are presented as the mean percentage of PMN adhesion to endothelial cells in relation to the untreated cells, which is assumed as $100 \%$ (Fig. 1A). A significant increase in the adhesion yield was observed after 6 hours of endothelial cell treatment with BK, compared to the untreated cells (by $40 \%$ ). On the contrary, simultaneous stimulation with both agonists led to total abolition of the BK-induced effect, and even a significant decrease in adhesion was observed (by $27 \%$, compared to the untreated cells). The prolonged stimulation of the cells with $\mathrm{BK}$ maintained the high level of PMN adhesion (an increase by 26\% and $13 \%$, after 12 hours and 24 hours, respectively). In the case of cells co-stimulated with BK and SUM for the longer periods (12 and 24 hours), PMN adhesion was significant increased (by $44 \%$ and $50 \%$, compared to the untreated cells for 12-h and 24-h incubation, respectively). The SUM-treated cells showed no changes regardless of the incubation time. 
A preincubation of endothelial cells with respective B2R and D2R antagonists, i.e. HOE 140 and eticlopride, confirmed a possible mediation of these receptors in the observed effects. For clearer presentation of the induced effects, the results from these experiments were presented as the average percentage of PMN adhesion to endothelial cells in comparison to different control samples: cells treated with BK at different lengths of incubation (Fig. 1B) and cells treated with BK+SUM, also at different incubation periods (Fig. 1C), were assigned with a value of $100 \%$. The treatment of the HOE 140-preincubated cells with $\mathrm{BK}$ resulted in a significant reduction of cell adhesion by $24 \%$ and $20 \%$ after 6 and 12 hours, respectively, compared to the corresponding control sample without the antagonist treatment. After 24 hours of incubation with $\mathrm{BK}$, the pretreatment with the $\mathrm{B} 2 \mathrm{R}$ antagonist did not change the effect induced by BK (Fig. 1B). In turn, the cell pretreatment with eticlopride did not significantly change the BK action (Fig. 1B). The most interesting observations are related to the results of the cells stimulated with BK+SUM after the pretreatment with the antagonists (Fig. 1C). In this case, the $\mathrm{B} 2 \mathrm{R}$ antagonist was able to invert the effect achieved by the cells after 6-h incubation with $\mathrm{BK}+\mathrm{SUM}$, showing that PMN adhered to HOE 140-preincubated endothelial cells more strongly (by 20\%) compared to cells stimulated with BK+SUM but without the antagonist pretreatment. After the longer incubation with the agonists, the pretreatment with HOE 140 caused only a slight reduction of neutrophil-endothelial adhesion (by $11 \%$ and $14 \%$ for $12-\mathrm{h}$ and $24-\mathrm{h}$ incubation, respectively). The D2R antagonist caused similar effects. Short stimulation of the eticlopride-pretreated cells with both agonists (6 hours) enhanced PMN adhesion (by 55\%); in turn, a significant decrease in this level (by $18 \%$ ) was achieved upon the longer stimulation with BK+SUM (24 hours). The use of antagonists did not cause any changes in the cells stimulated only with SUM (data not shown). Therefore, simultaneous activation of $\mathrm{D} 2 \mathrm{R}$ and $\mathrm{B} 2 \mathrm{R}$ may result in reduction of $\mathrm{BK}$-induced adhesion of leukocytes to endothelial cells. Nevertheless, this effect is dependent on the time of stimulation with agonists, showing that longer stimulation can invert the observed adhesion inhibition.

\section{B2R and D2R agonists modify neutrophil adhesion to cytokine-pretreated endothelial cells}

The adhesion of leukocytes to the endothelium is strictly associated with the action of inflammatory cytokines, including $\mathrm{TNF}-\alpha$. Since BK is a pro-inflammatory factor that can induce cytokine release in cells, in this study, we investigated how the effect induced by BK and SUM in "resting" cells can be disturbed when the cells were previously treated with TNF-a. We observed significant changes in the PMN adhesion to TNF- $\alpha$ preincubated endothelial cells after the treatment with the B2R and D2R agonists (Fig. 2). The results were presented as the change in cell adhesion after stimulation with the agonists in comparison to the values achieved in the non-stimulated cell at the corresponding incubation time. Cell adhesion was significantly enhanced when the endothelial cells were treated with $\mathrm{BK}$, and this effect was dependent on the incubation time (by 15\%, $16 \%$, and $68 \%$ for 6,12 and 24 hours, respectively). In turn, in contrast to the adhesion observed in the cytokine non-pretreated cell, SUM caused a slight but significant inhibition of neutrophil adhesion to endothelial cells. This effect was also dependent on the incubation

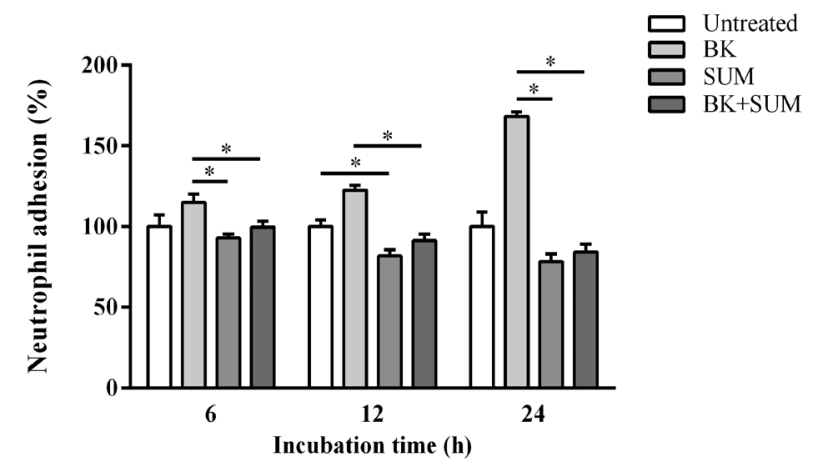

Figure 2. Neutrophil adhesion to TNF-a-treated endothelial cells after stimulation with B2R and D2R agonists.

Confluent cells were pretreated with $10 \mathrm{ng} / \mathrm{ml}$ TNF-a for 24 hours. After stimulation with $100 \mathrm{nM}$ BK, $100 \mathrm{nM}$ SUM, or both agonists simultaneously for 6,12 , and 24 hours, the cells were incubated with PMN labeled with fluorescence dye. The bars represent the mean values \pm S.D. calculated as the percentage of PMN adhesion to endothelial cells in relation to TNF- $\alpha$-stimulated cells, but not treated with the agonists, assumed to be $100 \%$. At least three experiments in duplicate were performed. ${ }^{*} p<0.05$ between the indicated samples.
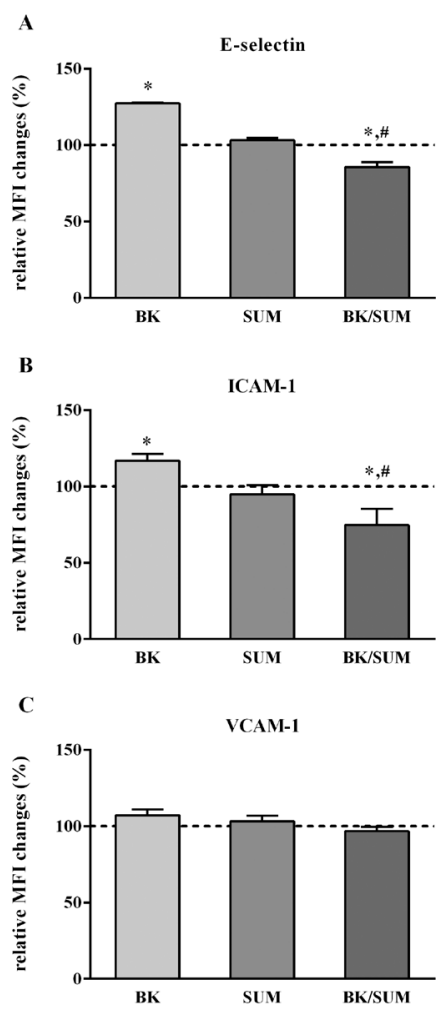

Figure 3. Adhesion proteins in endothelial cell membrane after stimulation with B2R and D2R agonists.

After 6-h stimulation with $100 \mathrm{nM} \mathrm{BK}, 100 \mathrm{nM}$ SUM, or both agonists simultaneously, confluent cells were analyzed by flow cytometry. Labeled antibodies to E-selectin (A), ICAM-1 (B), and VCAM-1 (C) were used. The values show the average percentage of changes in the mean fluorescence intensity (MFI) in comparison to the value obtained for the untreated cells, assumed to be $100 \%$. ${ }^{*} p<0.05$ versus untreated cells, ${ }^{*} p<0.05$ versus BK-treated cells.

time, showing the highest inhibition after 24 hours (by $8 \%, 9 \%$, and $22 \%$ for 6,12 , and 24 hours, respectively). The most unexpected results were obtained after incubation of the cells with BK+SUM. In these samples, the BK-induced effect on PMN adhesion was completely abolished, achieving even an inhibition of $16 \%$ below 


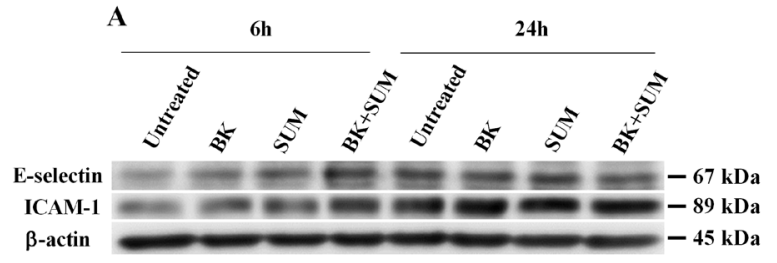

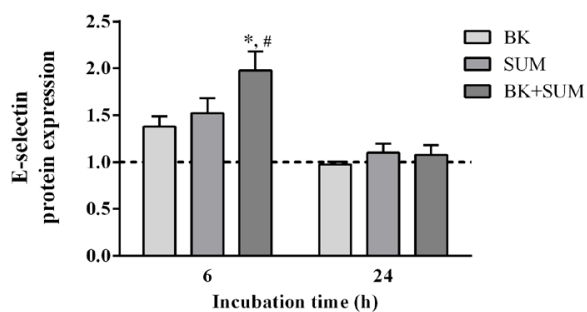

C

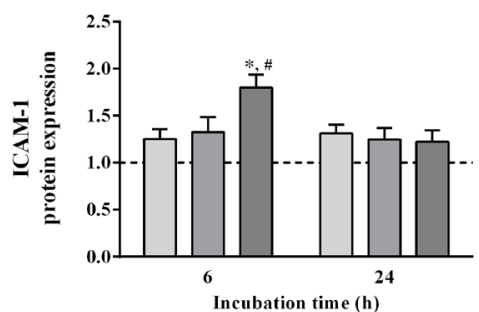

Figure 4. Effect of B2R and D2R agonists on the expression of E-selectin and ICAM-1 in endothelial cells.

Confluent cells were treated with $100 \mathrm{nM} \mathrm{BK}, 100 \mathrm{nM}$ SUM, or both agonists simultaneously for 6 or 24 hours. The level of adhesion protein expression was evaluated by Western blotting (A) and the bands obtained for E-selectin (B) and ICAM-1 (C) were analyzed by densitometry. The figures represent mean values \pm S.D. from two experiments, compared to the value obtained for the untreated cells assumed to be 1 (dashed line). ${ }^{*} p<0.01$ versus cells treated with $\mathrm{BK},{ }^{\#} p<0.01$ versus cells treated with SUM.

the value of untreated cells after 24 hours of incubation. Hence, this study clearly demonstrated a different response of activated endothelial cells when they are simultaneously incubated with selective agonists to B2R and D2R.

\section{Effect of BK and SUM on the expression of adhesion proteins in endothelial cells}

The adhesion of neutrophils to the endothelium implies rearrangement of various adhesion molecules in both neutrophils and endothelial cells. Here, we studied the effect of BK and SUM on the extracellular and total expression of the most important adhesion proteins in endothelial cells, i.e., E-selectin, ICAM-1, and VCAM-1. The flow cytometry studies allowed determining the presence of these proteins at the cell membrane (Fig. 3), whereas the total protein production was monitored with Western blotting (Fig. 4). The results from flow cytometry showed increased expression of E-selectin (Fig. 3A) and ICAM-1 (Fig. 3B) after 6h stimulation with BK, by $27 \%$ and $17 \%$, respectively, while no significant differences were noticed in VCAM-1 protein expression (Fig. 3C). In turn, the co-treatment with BK and SUM significantly inhibited the expression of E-selectin and ICAM-1 (by $15 \%$ and $25 \%$, respectively), but VCAM-1 expression did not change. On the other hand, the cell stimulation with SUM did not influence the expression of adhesion molecules in endothelial cells.

Since significant differences in PMN-endothelial cell adhesion were observed after 24-h incubation with BK and SUM, we evaluated the total production of E-selectin and ICAM-1 by endothelial cells induced with these two substances for different incubation periods. From Western blotting images two bands near 67 and $89 \mathrm{kDa}$ were detected, corresponding to E-selectin and ICAM-1 (Fig. 4A). These results indicated a significant time-dependent modification of the expression of these proteins in endothelial cells. The densitometric analysis of these bands was performed to calculate the protein content, which was normalized to the amount of $\beta$-actin protein. The resulted values were compared to those obtained for the untreated cells, which was assumed to be 1 . The expression of E-selectin (Fig. 4B) as well as ICAM-1 (Fig. 4C) significantly increased after $6 \mathrm{~h}$ incubation with BK. The values obtained for these samples were 1.37 and 1.25, respectively. Similarly, cells incubated with SUM exhibited higher amounts of both proteins, compared to the untreated cells, reaching a relative E-selectin and ICAM-1 expression level of 1.52 and 1.32, respectively. In the case of cells stimulated with BK+SUM simultaneously, the results for both proteins were strongly enhanced compared to the values observed after the treatment of endothelial cells with BK or SUM alone. In these samples, the calculated values were 1.97 and 1.79, respectively, for E-selectin and ICAM-1. The longer stimulation with the agonists caused slight changes in the ICAM-1 expression only $(1.31,1.24$, and 1.22 for the BK-, SUM-, and BK+SUM-treated cells, respectively). However, no significant differences were observed in the expression of E-selectin after the 24-h treatment with the agonists. Therefore, BK and SUM induced a quick production of adhesion molecules in the endothelial cells, which was significantly enhanced when the cells were simultaneously stimulated with both agonists. These effects were attenuated after longer stimulation.

\section{Effect of B2R and D2R agonists on interleukin-8 production by endothelial cells}

Interleukin-8, i.e. a chemotactic factor released from endothelial cells, attracts neutrophils during inflammatory processes. Hence, in this study, we also examined the role of BK and SUM in the regulation of IL- 8 production by endothelial cells. Indeed, cells that were not pretreated with $\mathrm{TNF}-\alpha$ did not show any significant changes in IL-8 production after the receptor agonist treatment (data not shown). However, in the TNF- $\alpha-$ stimulated cells, bradykinin caused increased IL-8 release, compared with the untreated cells (by 35\%), whilst reduced cytokine production was observed (by 33\%) in the SUM-treated cells (Fig. 5A). In turn, the cell stimulation with BK and SUM simultaneously led to total abolition of the BK- and SUM-induced effects, exhibiting values similar to those obtained for the untreated cells. To confirm the mediation of $\mathrm{B} 2 \mathrm{R}$ and $\mathrm{D} 2 \mathrm{R}$ in cytokine release, 
$\mathbf{A}$

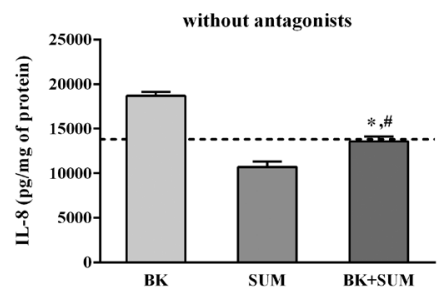

B

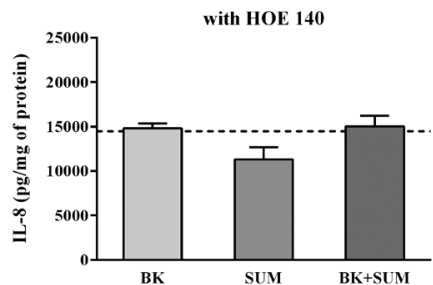

C

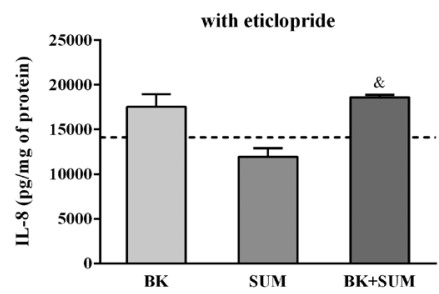

Figure 5. IL-8 release by TNF-a-treated endothelial cells after stimulation with B2R and D2R agonists.

Confluent cells were preincubated with $10 \mathrm{ng} / \mathrm{ml}$ TNF-a for 24 hours, followed by the stimulation with 100 nM BK, 100 nM SUM, or both agonists for 6 hours (A). Some samples were additionally pretreated with $10 \mu \mathrm{M}$ HOE 140 (B) or $10 \mu \mathrm{M}$ eticlopride (C) for 30 minutes before receptor agonist treatment. The production of IL-8 protein was determined by ELISA and normalized to the amount of protein in the samples. The value for the untreated cells is presented as a dashed line. The bars show the mean values \pm S.D. from at least three experiments in triplicate. ${ }^{*} p<0.05$ versus BKtreated cells, ${ }^{\prime} p<0.05$ versus SUM-treated cells, \& $p<0.05$ versus eticlopride/SUM-treated cells.

some experiments were also performed in cells pretreated with specific antagonists. HOE 140 inverted the BKinduced production of IL-8, but had no significant effect on the SUM- and BK+SUM-stimulated cells (Fig. 5B). In turn, eticlopride effectively inhibited the effect caused by $\mathrm{BK}+$ SUM-stimulation of cells, exhibiting a significant increase in IL-8 production (by $32 \%$, compared to the value obtained in cells treated only with eticlopride). No significant changes were observed in the BK- or SUMstimulated cells after the preincubation with the D2R antagonist (Fig. 5C). It should also be noted that the antagonists did not exert any important influence on the cytokine release by the endothelial cells. The IL-8 concentration in the untreated cells, regardless of antagonist preincubation, was comparable, reaching a mean value of $14142 \mathrm{pg} / \mathrm{mg}$ of protein.

\section{Co-treatment of endothelial cells with B2R and D2R agonists influences STAT3 phosporylation}

STAT3 is a crucial transcription factor involved in the regulation of cytokine production, including interleukin-8. In this study, we examined the activation of STAT3 by BK and SUM in endothelial cells, determining the protein expression of STAT3 phosphorylated at Tyr-705 and total STAT3 protein, with the Western blotting technique (Fig. 6A). The pSTAT3/STAT3 ratio was calculated after quantification of the corresponding
A

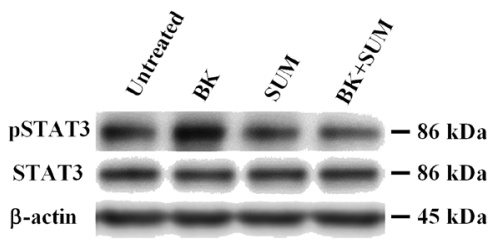

B

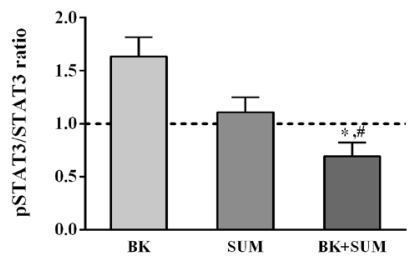

Figure 6. STAT3 activation in endothelial cells treated with B2R and D2R agonists.

After 15 min stimulation of confluent cells with $100 \mathrm{nM} \mathrm{BK}, 100 \mathrm{nM}$ SUM, or both agonists simultaneously for 15 minutes, the levels of pSTAT3 Tyr-705 and STAT3 protein expression were analyzed by Western blotting $(\mathbf{A})$. The pSTAT3/STAT3 ratio (B) was quantified by densitometric analysis and normalized to the amount of $\beta$-actin. The bars represent the mean values \pm S.D. from three independent experiments, which was compared to the value calculated for untreated cells, assumed to have a ratio equal to 1 (dashed line). ${ }^{*} p<0.01$ versus BK-treated cells, ${ }^{*} p<0.01$ versus SUMtreated cells.

bands by densitometric analysis. The values were normalized to the $\beta$-actin protein expression and the results were presented as a relative ratio in comparison to the value for the untreated cells, which was assumed to be 1 (Fig. 6B). The BK-stimulated cells showed a significant increase in STAT3 phosphorylation (by 63\%), whilst this ratio in the SUM-treated sample was similar to that in the untreated cells. Contrarily, the co-stimulation with $\mathrm{BK}$ and SUM reversed the BK-induced effect, showing even a significant decrease in the pSTAT3/STAT3 ratio compared to the untreated cells (by 31\%).

\section{DISCUSSION}

Numerous studies have confirmed that leukocyte adhesion to endothelial cells plays an important role in the pathogenesis of a variety of diseases. An imbalance between the production and degradation of proinflammatory stimuli causes uncontrolled leukocyte adhesion to the vascular wall leading to the development of atherosclerosis (Langer \& Chavakis, 2009). Our current study aimed to validate the possible role of the $B 2 R$ and $D 2 R$ in the regulation of neutrophil adhesion to endothelial cells. The analyzed immortalized endothelial cell line isolated from the human umbilical vein expresses both receptors endogenously, confirming earlier reports of the expression of these receptors in endothelial cells (Zarei et al., 2006; Terzuoli et al., 2014). In accordance with previous reports (Schuschke et al., 2001; Guevara-Lora et al., 2011), the current study indicated that BK increased the PMN adhesion to endothelial cells, with a maximal response observed after 6 hours of cell incubation (Fig. 1A). However, co-stimulation of the cells with SUM and BK abolished the BK-induced effect. The absence of SUM involvement in cell adhesion and the inversion of the BK-induced effect by $\mathrm{HOE} 140$, i.e. a B2R antagonist (Fig. 1B and $1 \mathrm{C}$ ), allow us to suggest that co-activation of $\mathrm{B} 2 \mathrm{R}$ and $\mathrm{D} 2 \mathrm{R}$ modified cell response due to a coop- 
eration between these receptors. Therefore, here we report for the first time on the protective role of D2R in the PMN adhesion to endothelial cells, induced by $\mathrm{BK}$, which is in agreement with previous studies demonstrating anti-inflammatory activity of this receptor (Zhang et al., 2015; Konkalmatt et al., 2016). Interestingly, the longer incubation produced an opposite effect, i.e. enhanced neutrophil adhesion after the co-treatment with BK and SUM. This observation may be associated with the emergence of other stimuli that can promote proinflammatory response, e.g. overexpression of B1R due to prolonged B2R stimulation (Guevara-Lora et al., 2009) or activation of signaling pathways promoting chemotactic activity (Koyama et al., 2000). Indeed, B1R mediation in the interaction between PMN and endothelial cells has been reported (Figueroa et al., 2015). The B1R agonist, Lys-des-Arg ${ }^{9}$ bradykinin, induced the expression of adhesion molecules in neutrophils and in endothelial cells, resulting in enhanced adhesiveness between cells. The observations resulting from experiments with the B2R antagonist make these assumptions more feasible, since the adhesiveness induced by $\mathrm{BK}$ is significantly decreased only at short incubation times. However, we cannot exclude an option that a growing amount of $\mathrm{B} 1 \mathrm{R}$ molecules can block the interaction between B2R and D2R. The dimerization between B2R and B1R has been demonstrated (Kang et al., 2004). This assumption makes our hypothesis more realistic, because the preincubation of the cells with the B2R and D2R antagonists did not reduce adhesion of cells stimulated with both agonists (Fig. 1C). Therefore, we summarize that diminished interactions between PMN and endothelial cells can be attributed to the cooperative action of B2R and D2R. In our study, we also analyzed the effect of BK and SUM on the PMN adhesion to endothelial cells that were previously activated with $\mathrm{TNF}-\alpha$, mimicking the situation in an inflamed tissue. The TNF- $\alpha$-preincubated cells showed significant changes in PMN adhesion to endothelial cells compared to that achieved in cells that were not activated with this cytokine, especially after the longer incubation with the agonists (Fig. 2). In this case, the longer incubation with both agonists, SUM and BK, strongly reduced the amount of PMN adhering to the endothelial cells, confirming the anti-inflammatory properties of the D2R agonist. Therefore, D2R activation may protect endothelial cells from imbalanced adhesion of leukocytes, even during severe inflammation.

Leukocyte adhesion to endothelial cells depends on the expression of adhesion molecules in the cell membrane. In this study, we observed that the BK-induced effect on PMN-endothelial adhesion was mainly related to an increase in the expression of E-selectin and ICAM-1 protein at the endothelial cell membrane (Fig. 3). These results agree with previous studies indicating the role of $\mathrm{BK}$ in the regulation of neutrophil rolling and sticking to endothelial cells through the induction of E-selectin and ICAM-1 expression (Shigematsu et al., 2002). As in the experiments performed on PMN-endothelial cell adhesion, SUM did not exert any significant effect on the level of adhesion molecule proteins. Nevertheless, a reduced amount of E-selectin and ICAM-1 was observed in the BK+SUM-treated cells, confirming that the D2R agonist downregulated the BKinduced effect. The role of D2R in the modulation of adhesion proteins has been poorly studied. To date, one recent study has shown that D2R silencing in mice after intracerebral hemorrhage may induce ICAM-1 expression (Zhang et al., 2015). Hence, the present study provides new facts on the D2R contribution to the regulation of the expression of adhesion proteins in endothelial cells. The absence of changes in VCAM-1 expression that can be related to lower constitutive expression of this protein in endothelial cells, compared to ICAM-1 or Eselectin, should be noted (Panes et al., 1999). The Western blotting results from samples treated with SUM or BK+SUM for 6 hours showed an opposite effect (Fig. 4). The total protein expression of E-selectin and ICAM1 significantly increased, which can be associated with enhanced production of proteins due to relocation to the cell membrane. Unexpectedly, we observed only a weak effect of the receptor agonists on the production of ICAM-1 after 24-h incubation. It should be emphasized that expression of adhesion proteins is dependent on the stimulation time and that the ICAM-1 expression is the most sustainable (Scholz et al., 1996; Panes et al., 1999). Therefore, we can assume that a balance between de novo protein synthesis and ICAM-1 relocation was achieved and that, at least after stimulation with $\mathrm{BK}+\mathrm{SUM}$, the increased amount of this protein may be responsible for the increased cell interaction (Fig. 1A).

The endothelial cells did not show any significant production of chemokine IL-8 after the stimulation with the $\mathrm{B} 2 \mathrm{R}$ and $\mathrm{D} 2 \mathrm{R}$ agonists (data not shown). However, the cell pretreatment with TNF- $\alpha$ facilitated detection of a varied influence of the $\mathrm{B} 2 \mathrm{R}$ and $\mathrm{D} 2 \mathrm{R}$ agonists on IL-8 release (Fig. 5). These results corroborate our suggestion that the specific D2R agonists can down-regulate uncontrolled adhesion of leukocytes to the endothelium induced by BK. However, we cannot exclude that increased B1R expression due to cytokine treatment may mediate cell adhesion. Of course, we observed that endothelial cells treated with TNF- $\alpha$ showed greater capacity to bind more amounts of neutrophils. Nevertheless, HOE 140 abolished the action of BK, which confirmed the concerted participation of B2R. Instead, the inhibition of IL-8 release achieved after the BK+SUM stimulation was not changed by HOE 140, but eticlopride reversed this effect, suggesting that the effect can be attributed to direct cooperation between B2R and D2R. Therefore, our study provides new information that can be helpful for better understanding of the modulation of adhesion processes in the vascular wall during inflammation.

Looking for mechanisms involved in the effects exerted by the co-activation of B2R and D2R on PMN adhesion to endothelial cells, we examined the mediation of the transcriptional factor STAT3. This protein modulates PMN adhesion to endothelial cells by controlling the expression of adhesion molecules (Kim et al., 2017; Wei et al., 2018) and interleukin-8 production (Gharavi et al., 2007). Previous reports have demonstrated an important role of BK in the induction of STAT3 phosphorylation in endothelial cells. Ju et al. (2000) reported an increased pSTAT3 level in bovine aortic endothelial cells 5 minutes after B2R activation. Similar results, with a maximal response after 15 minutes of BK-induction, were obtained in studies performed on a human microvascular endothelial cell line (Guevara-Lora et al., 2014). In this study, we demonstrated enhanced STAT3 phosphorylation after the 15 -min treatment with $\mathrm{BK}$, but it was significantly reduced after the co-stimulation with BK and SUM (Fig. 6). These observations correlate with the finding discussed above and corroborate the protective role of $\mathrm{D} 2 \mathrm{R}$ agonists in the inhibition of pro-inflammatory processes induced by $\mathrm{BK}$ in the vascular system. However, additional studies analyzing the B1R involvement should be conducted, since this receptor has a great importance for leukocyte adhesiveness during chronic inflammation 
(Figueroa et al., 2015) and is concurrently able to regulate STAT3 activation in monocytes (Guevara-Lora et al., 2014). Although the influence of D2R on the regulation of STAT3 phosphorylation has been reported (Kim et al., 2010; Qiu et al., 2016), our study showed no significant changes in the level of this parameter. However, these previous contrary studies were performed in nervous tissues. Nevertheless, our results provide new facts about the mechanism of adhesion modulation by D2R and B2R via STAT3 phosphorylation.

In conclusion, the data obtained in this study indicate an important role of $\mathrm{D} 2 \mathrm{R}$ in the regulation of the effects exerted by the B2R agonist on the inflammatory response of endothelial cells, especially in terms of the regulation of neutrophil adhesion to endothelial cells. These effects seem to be associated with a possible cooperation between these receptors, which can be involved in direct mutual interactions at the cell membrane, thus modifying their characteristic signaling pathways. The findings presented herein enrich the existing knowledge of the comprehensive functionality of the endothelium and may open up new perspectives in the search for modulators of $\mathrm{B} 2$ and $\mathrm{D} 2$ receptors to develop new drug candidates with potential therapeutic activity for treatment of cardiovascular disorders.

\section{Conflicts of Interest}

The authors declare that they have no conflicts of interests.

\section{Acknowledgements of Financial Support}

This study was supported in part by funds allocated to the Faculty of Biochemistry, Biophysics and Biotechnology, Jagiellonian University (Leading National Research Center (KNOW)) by the Ministry of Science and Higher Education (Scholarship no. BMN 8/2015 and No. BMN 11/2016, awarded to Ph.D. student A. N.-S.).

\section{REFERENCES}

Besser MJ, Ganor Y, Levite M (2005) Dopamine by itself activates either D2, D3 or D1/D5 dopaminergic receptors in normal human T-cells and triggers the selective secretion of either IL-10, TNFalpha or both. I Neuroimmunol 169: 161-171. https://doi.org/10.1016/j. jneuroim.2005.07.013

Bhoola K.D., Figueroa C.D., Worthy K. (1992). Bioregulation of kinins: kallikreins, kininogens and kininases. Pharmacol Rev 44: 1-80

Blais CJ, Marceau F, Rouleau JL, Adam A (2000) The kallikrein-kininogen-kinin system: lessons from the quantification of endogenous kinins. Peptides 21: 1903-1940. https://doi.org/10.1016/S01969781(00)00348-X

Ferre S, Casado V, Devi LA, Filizola M, Jockers R, Lohse MJ, Milligan G, Pin JP, Guitart X (2014) G protein-coupled receptor oligomerization revisited: functional and pharmacological perspectives. Pharmacol Rev 66: 413-434. http://dx.doi.org/10.1124/pr.113.008052

Figueroa CD, Matus CE, Pavicic F, Sarmiento J, Hidalgo MA, Burgos RA, Gonzalez CB, Bhoola KD, Ehrenfeld P (2015) Kinin B1 receptor regulates interactions between neutrophils and endothelial cells by modulating the levels of Mac-1, LFA-1 and intercellular adhesion molecule-1. Innate Immun 21: 289-304. http://dx.doi. org/10.1177/1753425914529169

Gharavi NM, Alva JA, Mouillesseaux KP, Lai C, Yeh M, Yeung W, Johnson J, Szeto WL, Hong L, Fishbein M, Wei L, Pfeffer LM, Berliner JA (2007) Role of the Jak/STAT pathway in the regulation of interleukin- 8 transcription by oxidized phospholipids in vitro and in atherosclerosis in vivo. I Biol Chem 282: 31460-31468. http:// dx.doi.org/10.1074/jbc.M704267200

Guevara-Lora I, Florkowska M, Kozik A (2009) Bradykinin-related peptides up-regulate the expression of kinin B1 and B2 receptor genes in human promonocytic cell line U937. Acta Biochim Pol 56: $515-522$

Guevara-Lora I, Labedz A, Skrzeczynska-Moncznik J, Kozik A (2011) Bradykinin and des-Arg10-kallidin enhance the adhesion of polymorphonuclear leukocyes to extracellular matrix proteins and en- dothelial cells. Cell Adhes Commun 18: 67-71. https://doi.org/10.31 09/15419061.2011.617854

Guevara-Lora I, Stalinska K, Augustynek B, Labedz-Maslowska A (2014) Influence of kinin peptides on monocyte-endothelial cell adhesion. I Cell Biochem 115: 1985-1995. http://dx.doi.org/10.1002/ jcb. 24870

Guevara-Lora I, Niewiarowska-Sendo A, Polit A, Kozik A (2016) Hypothetical orchestrated cooperation between dopaminergic and kinin receptors for the regulation of common functions. Acta Biochim Pol 63: 387-396. https://doi.org/10.18388/abp.2016_1366

Horke S, Witte I, Wilgenbus P, Kruger M, Strand D, Forstermann U (2007) Paraoxonase-2 reduces oxidative stress in vascular cells and decreases endoplasmic reticulum stress-induced caspase activation. Circulation 115: 2055-2064. https://doi.org/10.1161/CIRCULATIONAHA.106.681700

Ishihara K, Kamata M, Hayashi I, Yamashina S, Majima M (2002) Roles of bradykinin in vascular permeability and angiogenesis in solid tumor. Int Immunopharmacol 2: 499-509. https://doi.org/10.1016/ S1567-5769(01)00193-X

Ju H, Venema VJ, Liang H, Harris MB, Zou R, Venema RC (2000) Bradykinin activates the Janus-activated kinase/signal transducers and activators of transcription (JAK/STAT) pathway in vascular endothelial cells: localization of JAK/STAT signalling proteins in plasmalemmal caveolae. Biochem J 351: 257-264. https://doi. org/10.1042/bj3510257

Kang DS, Ryberg K, Morgelin M, Leeb-Lundberg LMF (2004) Spontaneous formation of a proteolytic B1 and B2 bradykinin receptor complex with enhanced signaling capacity. J Biol Chem 279: 2210222107. http://doi.org/10.1074/ibc.M402572200

Kim KS, Yoon YR, Lee HJ, Yoon S, Kim SY, Shin SW, An JJ, Kim MS, Choi SY, Sun W, Baik JH (2010) Enhanced hypothalamic leptin signaling in mice lacking dopamine D2 receptors. I Biol Chem 285: 8905-8917. https://doi.org/10.1074/jbc.M109.079590

Kim KJ, Kwon SH, Yun JH, Jeong HS, Kim HR, Lee EH, Ye SK, Cho CH (2017) STAT3 activation in endothelial cells is important for tumor metastasis via increased cell adhesion molecule expression. Oncogene 36: 5445-5459. https://doi.org/10.1038/onc.2017

Konkalmatt PR, Asico LD, Zhang Y, Yang Y, Drachenberg C, Zheng X, Han F, Jose PA, Armando I (2016) Renal rescue of dopamine D2 receptor function reverses renal injury and high blood pressure. JCI Insight 1: e85888. https://doi.org/10.1172/jci.insight.85888

Koyama S, Sato E, Numanami H, Kubo K, Nagai S, Izumi T (2000) Bradykinin stimulates lung fibroblasts to release neutrophil and monocyte chemotactic activity. Am J Respir Cell Mol Biol 22: 75-84. https://doi.org/10.1165/ajrcmb.22.1.3752

Langer HF, Chavakis T (2009) Leukocyte-endothelial interactions in inflammation. J Cell Mol Med 13: 1211-1220. https://doi.org/10.1111/ j.1582-4934.2009.00811.x

Leeb-Lundberg LM, Marceau F, Muller-Esterl W, Pettibone DJ, Zuraw BL (2005) International union of pharmacology. XLV. Classification of the kinin receptor family: from molecular mechanisms to pathophysiological consequences. Pharmacol Rev 57: 27-77. https:// doi.org/10.1124/pr.57.1.2

Lehmberg J, Beck J, Baethmann A, Uhl E (2003) Bradykinin antagonists reduce leukocyte-endothelium interactions after global cerebral ischemia. I Cereb Blood Flow Metab 23: 441-448. https://doi. org/10.1097/01.WCB.0000052280.23292.35

McCall RB, Lookingland KJ, Bedard PJ, Huff RM (2005) Sumanirole, a highly dopamine D2-selective receptor agonist: in vitro and in vivo pharmacological characterization and efficacy in animal models of Parkinson's disease. J Pharmacol Exp Ther 314: 1248-1256. https:// dx.doi.org/10.1124/jpet.105.084202

Mestas J, Ley K (2008) Monocyte- endothelial cell interactions in the development of atherosclerosis. Trends Cardiovasc Med 18: 228-232. https://dx.doi.org/10.1016/j.tcm.2008.11.004

Missale C, Nash SR, Robinson SW, Jaber M Caron MG (1998) Dopamine receptors: from structure to function. Physiol Rev 78: 189-225. https://dx.doi.org/10.1152/physrev.1998

Niewiarowska-Sendo A, Polit A, Piwowar M, Tworzydło M, Kozik A, Guevara-Lora I (2017) Bradykinin B2 and dopamine D2 receptors form a functional dimer. Biochim Biophys Acta 1864: 1855-1866. https://doi.org/10.1016/j.bbamcr.2017.07.012

Panes J, Perry M, Granger DN (1999) Leukocyte-endothelial cell adhesion: avenues for therapeutic intervention. Br I Pharmacol 126: 537550. https://doi.org/10.1038/sj.bjp.0702328

Qiu J, Yan Z, Tao K, Li Y, Li Y, Li J, Dong Y, Feng D, Chen H (2016) Sinomenine activates astrocytic dopamine D2 receptors and alleviates neuroinflammatory injury via the CRYAB/STAT3 pathway after ischemic stroke in mice. I Neuroinflammation 13: 263. https:// doi.org/10.1186/s12974-016-0739-8

R Core Team (2016) R: A language and environment for statistical computing, R Foundation for Statistical Computing, Vienna, Austria. https://www.r-project.org/

Roscioni SS, Kistemaker LE, Menzen MH, Elzinga CR, Gosens R,Halayko AJ, Meurs H, Schmidt M (2009) PKA and Epac cooperate to augment bradykinin-induced interleukin- 8 release from 
human airway smooth muscle cells. Respir Res 10: 88. https://doi. org/10.1186/1465-9921-10-88

Scholz D, Devaux B, Hirche A, Pötzsch B, Kropp B, Schaper W, Schaper J (1996) Expression of adhesion molecules is specific and time-dependent in cytokine-stimulated endothelial cells in culture. Cell Tissue Res. 284: 415-423

Schuschke DA, Saari JT, Miller FN (2001) Leukocyte-endothelial adhesion is impaired in the cremaster muscle microcirculation of the copper-deficient rat. Immunol Lett 76: 139-144. https://doi. org/10.1016/s0165-2478(01)00171-7

Shigematsu S, Ishida S, Gute DC, Korthuis RJ (2002) Bradykinininduced proinflammatory signaling mechanisms. Am $J$ Physiol Heart Circ Physiol 283: H2676-H2686. https://doi.org/10.1152/ajpheart.00538.2002

Su JB (2015) Vascular endothelial dysfunction and pharmacological treatment. World J Cardiol 7: 719-741. https://doi.org/10.4330/wjc. v7.i11.719

Terzuoli E, Meini S, Cucchi P, Catalani C, Cialdai C, Maggi CA, Giachetti A, Ziche M, Donnini S (2014) Antagonism of bradykinin B2 receptor prevents inflammatory responses in human endothelial cells by quenching the NF-kB pathway activation. PLoS One 9: e84358. https://doi.org/10.1371/journal.pone.0084358
Wei Z, Jiang W, Wang H, Li H, Tang B, Liu B, Jiang H, Sun X (2018) The IL-6/STAT3 pathway regulates adhesion molecules and cytoskeleton of endothelial cells in thromboangiitis obliterans. Cell Signal 44: 118-126. https://doi.org/10.1016/j.cellsig.2018.01.015

Zarei S, Frieden M, Rubi B, Villemin P, Gauthier BR, Maechler P, Vischer UM (2006) Dopamine modulates von Willebrand factor secretion in endothelial cells via D2-D4 receptors. J Thromb Haemost 4: 1588-1595. https://doi.org/10.1111/j.1538-7836.2006.01998.x

Zawrotniak M, Bochenska O, Karkowska-Kuleta J, Seweryn-Ozog K, Aoki W, Ueda M, Kozik A, Rapala-Kozik M (2017) Aspartic proteases and major cell wall components in Candida albicans trigger the release of neutrophil extracellular traps. Front Cell Infect Microbiol 7: 414. https://doi.org/10.3389/fcimb.2017.00414

Zhang Y, Cuevas S, Asico LD, Escano C, Yang Y, Pascua AM, Wang X, Jones JE, Grandy D, Eisner G, Jose PA, Armando I (2012) Deficient dopamine D2 receptor function causes renal inflammation independently of high blood pressure. PLoS One 7: e38745. https:// doi.org/10.1371/journal.pone.0038745

Zhang Y, Chen Y, Wu J, Manaenko A, Yang P, Tang J, Fu W, Zhang JH (2015) Activation of dopamine D2 receptor suppresses neuroinflammation through $\alpha \mathrm{B}$-crystalline by inhibition of NF- $x \mathrm{~B}$ nuclear translocation in experimental ICH mice model. Stroke 46: 26372646. https://doi.org/10.1161/STROKEAHA.115.009792 